\title{
Genome sequences of Arthrobacter spp. that use a modified sulfoglycolytic Embden-Meyerhof-Parnas pathway
}

\author{
Arashdeep Kaur ${ }^{1,2}$. Phillip L. van der Peet ${ }^{1,2}$. Janice W.-Y. Mui ${ }^{1,2} \cdot$ Marion Herisse $^{3} \cdot$ Sacha Pidot $^{3}$. \\ Spencer J. Williams ${ }^{1,2}$ (D)
}

Received: 7 December 2021 / Revised: 3 February 2022 / Accepted: 11 February 2022 / Published online: 24 February 2022

(c) The Author(s) 2022

\begin{abstract}
Sulfoglycolysis pathways enable the breakdown of the sulfosugar sulfoquinovose and environmental recycling of its carbon and sulfur content. The prototypical sulfoglycolytic pathway is a variant of the classical Embden-Meyerhof-Parnas (EMP) pathway that results in formation of 2,3-dihydroxypropanesulfonate and was first described in gram-negative Escherichia coli. We used enrichment cultures to discover new sulfoglycolytic bacteria from Australian soil samples. Two gram-positive Arthrobacter spp. were isolated that produced sulfolactate as the metabolic end-product. Genome sequences identified a modified sulfoglycolytic EMP gene cluster, conserved across a range of other Actinobacteria, that retained the core sulfoglycolysis genes encoding metabolic enzymes but featured the replacement of the gene encoding sulfolactaldehyde (SLA) reductase with SLA dehydrogenase, and the absence of sulfoquinovosidase and sulfoquinovose mutarotase genes. Excretion of sulfolactate by these Arthrobacter spp. is consistent with an aerobic saprophytic lifestyle. This work broadens our knowledge of the sulfo-EMP pathway to include soil bacteria.
\end{abstract}

Keywords Sulfur cycle $\cdot$ Enrichment $\cdot$ Nuclear magnetic resonance spectroscopy $\cdot$ Isotope labeling

Abbreviations
$\begin{array}{ll}\text { ABC } & \text { ATP-binding cassette } \\ \text { DHPS } & \text { 2,3-Dihydroxypropanesulfonate } \\ \text { NAD(P)H } & \begin{array}{l}\text { Reduced nicotinamide adenine dinucleotide } \\ \text { (phosphate) }\end{array} \\ \text { SF } & \text { Sulfofructose } \\ \text { SL } & \text { Sulfolactate } \\ \text { SLA } & \text { Sulfolactaldehyde } \\ \text { SQ } & \text { 6-Deoxy-6-sulfo-D-glucose } \\ \text { SQase } & \text { Sulfoquinovosidase } \\ \text { SQDG } & \text { Sulfoquinovosyl diacylglycerol } \\ \text { SQGro } & \text { Sulfoquinovosyl glycerol }\end{array}$

Communicated by Johann Heider.

Spencer J. Williams

sjwill@unimelb.edu.au

1 School of Chemistry, University of Melbourne, Parkville, VIC 3010, Australia

2 Bio21 Molecular Science and Biotechnology Institute, University of Melbourne, Parkville, VIC 3010, Australia

3 Department of Microbiology and Immunology, University of Melbourne, Peter Doherty Institute for Infection and Immunity, Melbourne, VIC 3000, Australia
Sulfo-ED Sulfoglycolytic Entner-Doudoroff

Sulfo-EMP Sulfoglycolytic Embden-Meyerhof-Parnas

Sulfo-SFT Sulfoglycolytic sulfofructose transaldolase

Sulfo-TK Sulfoglycolytic transketolase

Sulfo-SMO Sulfoglycolytic sulfoquinovose monooxygenase

TSUP 4-Toluene sulfonate uptake permease

\section{Introduction}

Sulfoquinovose (SQ; 6-deoxy-6-sulfo-D-glucose) is a sulfosugar produced by photosynthetic organisms (GoddardBorger and Williams 2017). It is primarily found as the headgroup of the sulfoglycolipid sulfoquinovosyl diacylglycerol (SQDG) in photosynthetic tissues and membranes in plants, algae and cyanobacteria (Benson et al. 1959; Goddard-Borger and Williams 2017). The annual global production of SQ is estimated at $10^{10}$ tons per annum (Harwood and Nicholls 1979), and thus, the degradation of this sulfosugar is an important arm of the global biogeochemical cycle. The degradation of sulfoquinovose occurs through pathways of sulfoglycolysis and provides access to its constituent carbon and generates ATP and reducing equivalents (NADH/ 
NADPH) (Benson and Shibuya 1961; Snow et al. 2021). The sulfoglycolytic Embden-Meyerhof-Parnas (sulfo-EMP) (Denger et al. 2014; Sharma et al. 2021), Entner-Doudoroff (sulfo-ED) (Felux et al. 2015), and sulfofructose transaldolase (sulfo-SFT) (Frommeyer et al. 2020; Liu et al. 2020) pathways cleave the 6-carbon chain of SQ into two 3-carbon fragments, one of which is utilized in primary metabolism, while the other containing the sulfonate group is converted to either sulfolactate (SL) or 2,3-dihydroxypropanesulfonate (DHPS) and excreted. The sulfoglycolytic sulfofructose transketolase (sulfo-TK) pathway uses four of the six carbons in primary metabolism while the last two are excreted as isethionate (Liu et al. 2021). The sulfoglycolytic sulfoquinovose monooxygenase (sulfo-SMO) pathway results in cleavage of the $\mathrm{C}-\mathrm{S}$ bond of sulfoquinovose and leads to production of glucose, and thus enables the complete breakdown of the SQ molecule (Liu et al. 2021; Sharma et al. 2022). The genes encoding these sulfoglycolytic pathways are found within clusters that typically contain genes encoding proteins for the import of SQ or its glycosides, the export of the end-products, SL or DHPS, and a specialized glycoside hydrolase termed a sulfoquinovosidase (SQase) (Speciale et al. 2016; Abayakoon et al. 2018a; Liu et al. 2021) that can cleave SQ glycosides to release SQ that can undergo sulfoglycolysis.

We report here the use of sequential enrichment culturing using minimal media containing SQ as sole carbon source to isolate new sulfoglycolytic bacteria from soil. We identify two Arthrobacter sp. strains, AK01 and AK04, and demonstrate that they grow on SQ and secrete SL into the growth media. We present the draft genome sequences of AK01 and AK04 that reveals that these Arthrobacter sp. contain a gene cluster encoding a sulfoglycolytic Embden-Meyerhof-Parnas pathway that differs from the prototypical pathway of $E$. coli through the lack of an identifiable candidate SQase, the replacement of SLA reductase with SLA dehydrogenase, and the presence of $\mathrm{ABC}$ transporters and TauE permeases, variations that are present within other sequenced Actinobacteria.

\section{Materials and methods}

\section{Bacterial growth media}

Growth media were prepared using M9 minimal salts media $(2 \mathrm{~mL})$, trace metal solution $(0.1 \mathrm{~mL})$, and vitamin solution $(0.01 \mathrm{~mL})$ and contained $5 \mathrm{mM}$ sulfoquinovose (SQ) as sole carbon source, made up to a final volume of $10 \mathrm{~mL}$ with water. $\mathrm{M} 9$ minimal salts media contain $0.45 \mathrm{M} \mathrm{Na}_{2} \mathrm{HPO}_{4}$, $0.11 \mathrm{M} \mathrm{KH}_{2} \mathrm{PO}_{4}, 0.09 \mathrm{M} \mathrm{NH}_{4} \mathrm{Cl}, 0.04 \mathrm{M} \mathrm{NaCl}, 0.1 \mathrm{M}$ $\mathrm{MgSO}_{4}, 0.1 \mathrm{M} \mathrm{CaCl}_{2}$; trace metal solution contains $0.4 \mathrm{mM}$ $\mathrm{FeCl}_{3}, 0.08 \mathrm{mM} \mathrm{CoCl}_{2}, 0.08 \mathrm{mM} \mathrm{CuCl}_{2}, 0.08 \mathrm{mM} \mathrm{MnCl}_{2}$,
$0.55 \mathrm{mM} \mathrm{ZnCl}_{2}, 0.01 \mathrm{mM} \mathrm{NiCl}_{2}, 0.05 \mathrm{mM} \mathrm{Na}_{2} \mathrm{MoO}_{4}$ and $0.5 \mathrm{mM} \mathrm{H}_{3} \mathrm{BO}_{3}$; vitamin mixture contains $0.04 \mathrm{mM}$ biotin, $0.05 \mathrm{mM}$ calcium pantothenate, $0.15 \mathrm{mM}$ thiamine hydrochloride, $0.36 \mathrm{mM} p$-aminobenzoic acid, $0.81 \mathrm{mM}$ nicotinic acid, $1.49 \mathrm{mM}$ pyridoxamine dihydrochloride, $0.01 \mathrm{~B} 12$ (cyanocobalamin).

\section{Isolation of Arthrobacter sp.}

Arthrobacter sp. strains AK01 and AK04 were isolated from enriched culture, obtained from soil of the Botany Systems Garden (University of Melbourne).

Two soil samples were collected and approximately $1 \mathrm{~g}$ of soil was suspended in $5 \mathrm{~mL}$ of sterilized growth media containing $5 \mathrm{mM}$ SQ as a sole carbon source. The culture was incubated at $30{ }^{\circ} \mathrm{C}$ for 4 days with agitation at $250 \mathrm{rpm}$. A subsample $(100 \mu \mathrm{L})$ was transferred into fresh vitaminsupplemented M9 media and grown for a further 4 days. This step was repeated four times and after outgrowth of the final culture for 4 days, cells were plated onto LB agar plates (10 g/L tryptone, $5 \mathrm{~g} / \mathrm{L} \mathrm{NaCl}, 5 \mathrm{~g} / \mathrm{L}$ yeast, $15 \mathrm{~g} / \mathrm{L}$ agar) and incubated overnight at $30{ }^{\circ} \mathrm{C}$ in dark. Single colonies were picked and inoculated into fresh vitamin-supplemented M9 media containing $5 \mathrm{mM} \mathrm{SQ}$ and incubated at $28{ }^{\circ} \mathrm{C}$ while shaking at $250 \mathrm{rpm}$ using a Ratek orbital mixer incubator. Once the cultures were visibly turbid, cells were again plated onto LB agar, incubated overnight at $30^{\circ} \mathrm{C}$ in dark and single colonies picked and inoculated again into fresh vitamin-supplemented M9 media containing $5 \mathrm{mM}$ SQ. Once cultures were visibly turbid, frozen stocks were prepared by diluting to $10 \%$ glycerol and freezing at $-80{ }^{\circ} \mathrm{C}$. Cell morphology was examined using scanning electron microscopy. Genomic DNA was isolated using the GenElute DNA extraction kit (Sigma) with inclusion of lysozyme and RNAase.

\section{Phenotypic assays}

Bacteria were grown in vitamin-supplemented M9 minimal media containing ${ }^{13} \mathrm{C}_{6}$-SQ $(7.7 \mathrm{mM})$ in culture tubes at $30{ }^{\circ} \mathrm{C}$ for 1 day with agitation at $250 \mathrm{rpm}$. After cultures became visibly turbid, the cells were sedimented by centrifugation at $9000 \mathrm{~g}$ for $10 \mathrm{~min}$ (using Sigma laborzentrifugen model $1-15$, rotor 12124) and the supernatant was diluted with 50\% $\mathrm{D}_{2} \mathrm{O}$ and transferred to a $5 \mathrm{~mm}$ NMR tube. ${ }^{13} \mathrm{C}$-NMR spectra were acquired using a $500 \mathrm{MHz}$ instrument and are shown in Fig. 1a.

\section{Genome sequence, assembly, and annotation}

Genomic DNA was sequenced using an Illumina NextSeq at the Peter Doherty Institute for Infection and Immunity, Parkville, Victoria, Australia. DNA was prepared for 


\section{a AK01}

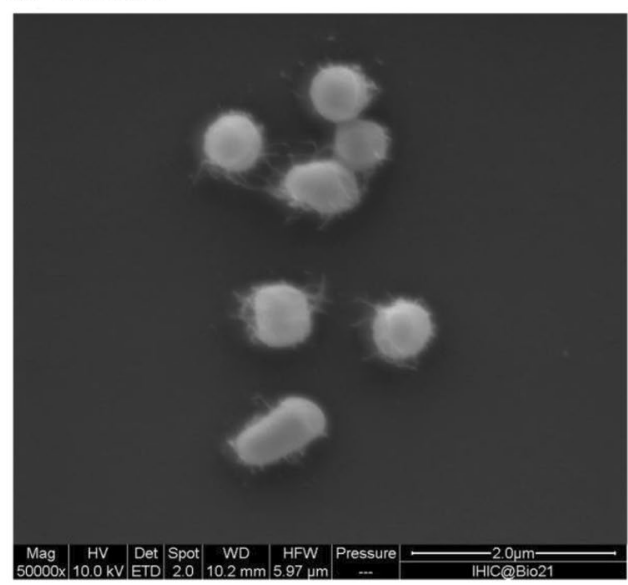

\section{b AK04}

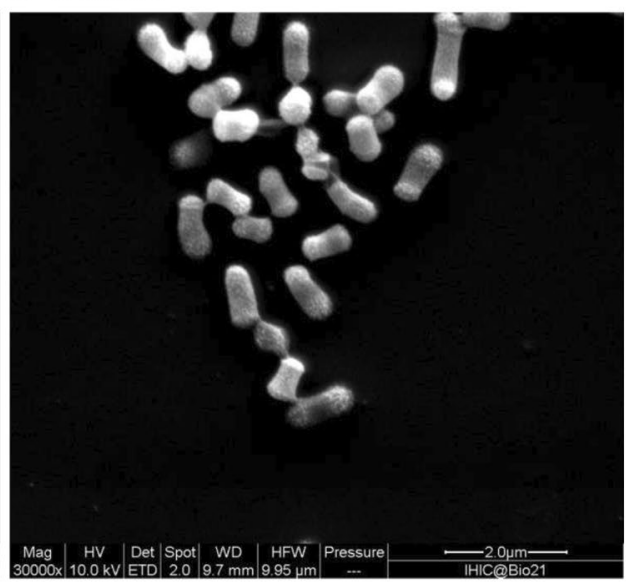

Fig. 1 Scanning electron microscopy image of Arthrobacter sp. a strain AK01, b strain AK04. Cell morphology was examined using a scanning electron microscope (Quanta 200 ESEM). Cells were grown in LB media for 3 days, fixed in $0.05 \%$ glutaraldehyde in $0.1 \mathrm{M}$ phosphate buffer ( $\mathrm{pH} 7.4$ ), then in $2.5 \%$ glutaraldehyde in $0.1 \mathrm{M}$ phosphate buffer $(\mathrm{pH}$ 7.4) and allowed to react for $20 \mathrm{~min}$.

Table $1{ }^{13} \mathrm{C}$-NMR $(125 \mathrm{MHz})$ data of ${ }^{13} \mathrm{C}_{3}$-SL produced as metabolite from ${ }^{13} \mathrm{C}_{6}$-SQ

\begin{tabular}{llll}
\hline $\begin{array}{l}\text { Chemical } \\
\text { shift }(d, \mathrm{ppm})\end{array}$ & Multiplicity & Coupling constant $(\mathrm{Hz})$ & Assignment \\
\hline 55.19 & $\mathrm{~d}$ & ${ }^{1} J_{\mathrm{C} 1, \mathrm{C} 2}=36.9$ & $\mathrm{C} 3$ \\
62.42 & $\mathrm{~s}$ & $\mathrm{NA}$ & - \\
68.85 & $\mathrm{dd}$ & ${ }^{1} J_{\mathrm{C} 2, \mathrm{C} 3}=52.7$ & $\mathrm{C} 2$ \\
& & ${ }^{1} J_{\mathrm{C} 1, \mathrm{C} 2}=38.0$ & \\
72.01 & $\mathrm{~s}$ & $\mathrm{NA}$ & - \\
178.69 & $\mathrm{~d}$ & ${ }^{1} J_{\mathrm{C} 2, \mathrm{C} 3}=54.6$ & $\mathrm{C} 1$ \\
\hline
\end{tabular}

Samples contain $50 \% \mathrm{D}_{2} \mathrm{O}$ to allow frequency lock

sequencing on the Illumina NextSeq platform using the Nextera XT DNA preparation kit (Illumina) with $\times 150 \mathrm{bp}$ paired end chemistry and with a targeted sequencing depth of $>50 \times$. Draft genomes were assembled using Shovill v1.1.0 (https://github.com/tseemann/shovill) and annotated using Prokka v1.14.5 (Seemann 2014). GC percentage and ANI calculations were performed using ANI calculator (https://www.ezbiocloud.net/tools/ani) (Yoon et al. 2017). Assembled genomes have been deposited at the NCBI (GenBank accession: AK01, SAMN23041292; AK04, SAMN23041293). General features for isolated bacteria are reported in Table 1. The protein sequences of putative sulfoglycolysis proteins in AK01 and AK04 were used to search against the NCBI non-redundant database using BLASTp. Percentage identities for key sulfo proteins are given in Table $\mathrm{S} 2$.
Fixed cells were adhered onto poly-lysine-coated slides and rinsed with water 3 times, then dehydrated by soaking in an ascending ethanol gradient (20-100\%). The sample was critical point dried using a Leica CPD3000 and gold coated to thickness of $5 \mathrm{~nm}$ using Safematic CCU-010 compact coating unit. Images are at approximately $50,000 \times$ magnification with scale bar shown

\section{Discovery of related sulfoglycolytic operons}

Sequences for $E$. coli sulfoquinovosidase (NP_418314.1, locus tag b3878), SQ mutarotase (NP_418315.3, locus tag b3879), SQ isomerase (NP_418316.4, locus tag b3880), SF kinase (NP_418319.2, locus tag b3883), SFP aldolase (NP_418317.1, locus tag b3881), SLA reductase (NP_418318.1, locus tag b3882) and sulfo-EMP regulator (NP_418320.2, locus tag b3884) were submitted separately as queries to the NCBI BLASTp tool. The database searched was the non-redundant protein sequence (nr) database, with E. coli (taxid: 562) sequences excluded. Standard algorithm parameters were used, except the maximum target sequences was set to 10,000. The results were filtered, with only protein sequences with $E$-value $\leq 5.41 \mathrm{e}-44$ retained. The corresponding nucleotide accession numbers for each protein from all seven searches were extracted, and the seven lists combined and duplicates removed to give a list of candidate genome sequences. This list was converted into a MultiGeneBLAST reference library and searched using the E. coli sulfo-EMP gene cluster as a query. Scripts for this pipeline are available on GitHub (https:// github.com/jmui-unimelb/Gene-Cluster-Search-Pipeline). Gene clusters found using this workflow were screened for clusters that contained a putative SQ isomerase, a putative SF kinase and a putative SFP aldolase, but lacked a homologous sulfoquinovosidase. 


\section{Results and discussion}

\section{Isolation and characterization of sulfoglycolytic bacteria}

Bacteria able to grow on SQ as sole carbon source were selected by using soil samples (collected from the University of Melbourne, Parkville campus) to inoculate a vitamin-enriched minimal media containing SQ as sole carbon source. Sequential subculturing into fresh SQ-minimal media, followed by plating onto LB agar and then regrowth in SQ-minimal media led to isolation of strains AK01 and AK04 that possessed a short rod-like appearance (Fig. 1). Strains AK01 and AK04 grew robustly on SQ with peak growth rates of 0.129 and $0.081 \mathrm{~A}_{600} / \mathrm{min}$ and achieved stationary phase after approximately 25 and
40 h, respectively (Fig. 2a, c). The absorbance at stationary phase for cultures grown on SQ were approximately half of that for cultures grown on equimolar glucose. ${ }^{13} \mathrm{C}-\mathrm{NMR}$ analysis of culture medium of AK01 and AK04 grown on ${ }^{13} \mathrm{C}_{6}$-SQ (7.7 mM) media gave three signals corresponding to ${ }^{13} \mathrm{C}_{3}$-SL (Fig. 2b, d, Table 1). ${ }^{13} \mathrm{C}$-NMR analysis of spent culture confirmed that substrate SQ is completely consumed by bacteria and metabolized to SL.

To investigate the genetic basis for SQ consumption by AK01 and AK04, DNA extracted from these bacteria were sequenced using the Illumina NextSeq platform. Table 2 shows the key features of the two draft genomes. On the basis of 16S rRNA gene sequence analysis the organisms were assigned as Arthrobacter sp. The 16S rRNA genes of the two strains were $98.8 \%$ identical over $1520 \mathrm{bp}$, which suggests that these organisms are of the same species (Kim et al. 2014). However, average nucleotide identity (ANI)

a Arthrobactersp. (AK04)

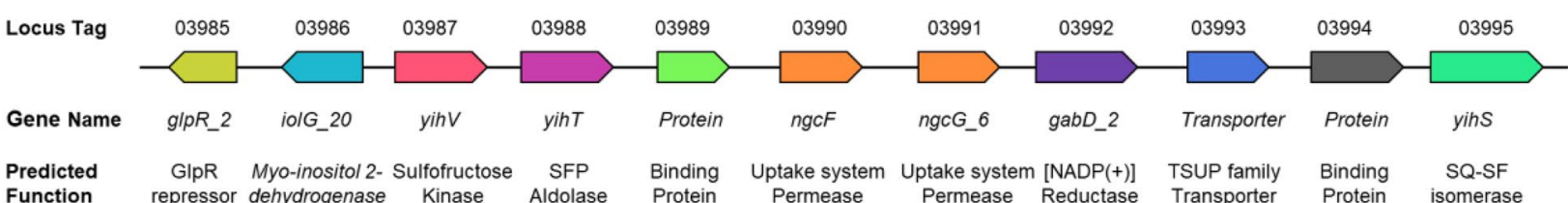

b Arthrobacter sp. (AK01)

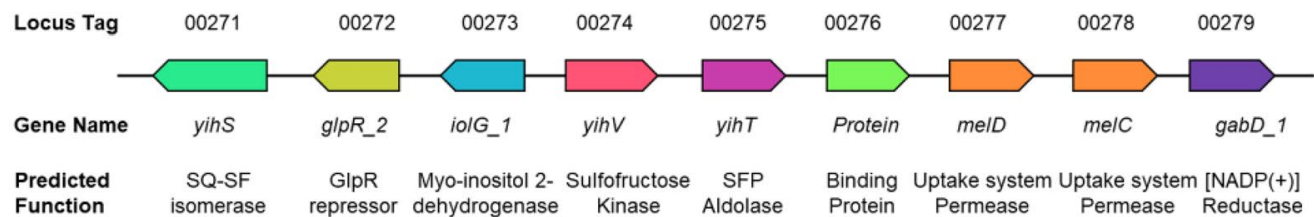

c Arthrobacterspp.

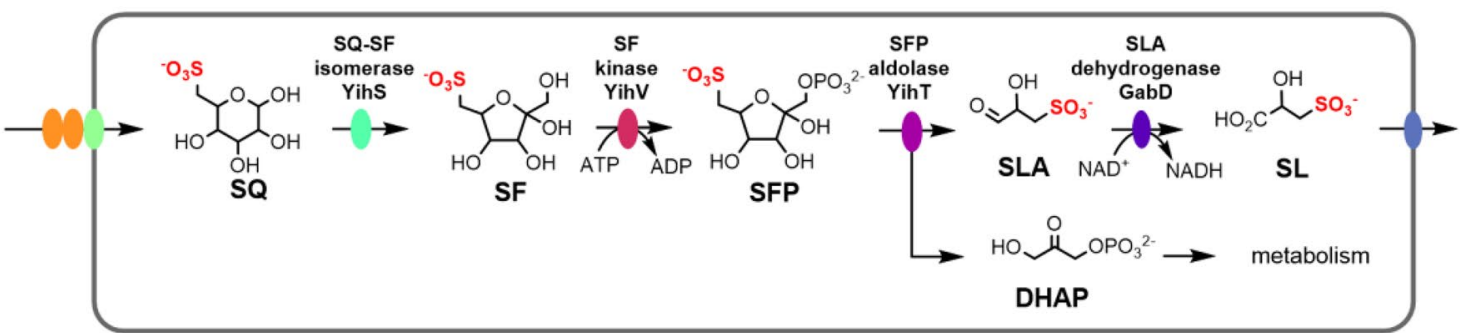

d E. coli K12

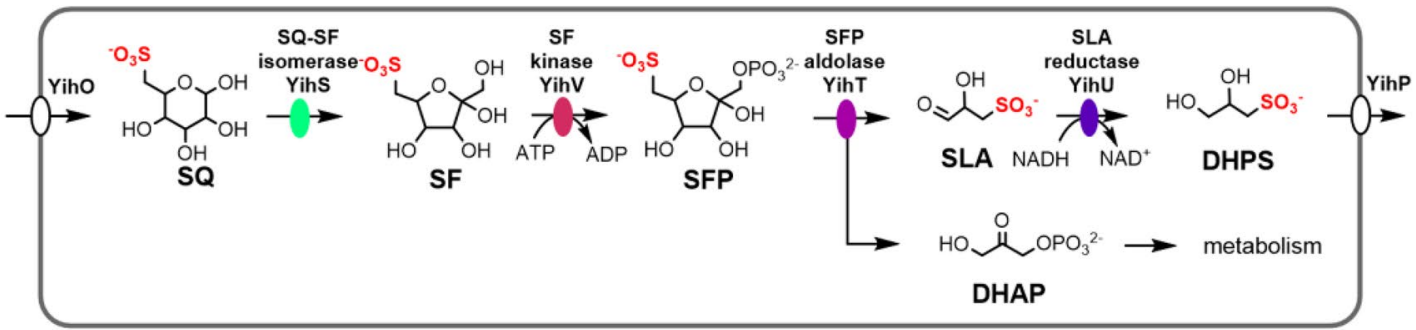

Fig. 2 Proposed sulfoglycolytic Embden-Meyerhof-Parnas (sulfoEMP) pathway for Arthrobacter spp. a Gene cluster encoding the sulfo-EMP pathway for Arthrobacter sp. AK04. b Gene cluster encoding the sulfo-EMP pathway for Arthrobacter sp. AK01. c Proposed sulfo-EMP pathway for Arthrobacter spp. d Comparison with EMP pathway for E. coli $\mathrm{K} 12$ 
Table 2 Key features of the AK01 (GenBank accession: SAMN23041292) and AK04 (GenBank accession: AK01, SAMN23041293) draft genome assemblies

\begin{tabular}{lll}
\hline & AK01 & AK04 \\
\hline Genome size & $5,105,913$ & $4,700,363$ \\
Number of contigs & 169 & 130 \\
GC \% & 62.9 & 65.5 \\
Number of ORFs & 4734 & 4318 \\
Number of putative genes & 4669 & 4261 \\
Number of putative tRNA & 59 & 53 \\
Number of putative rRNA & 5 & 3 \\
Number of putative tmRNA & 1 & 1 \\
Number of genes assigned a func- & $2478(53 \%)$ & $2219(52 \%)$ \\
$\quad$ tion (\%) & & \\
\hline
\end{tabular}

between the two organisms is only $76.5 \%$, conflicting with the 16S rDNA gene results and suggesting that these two organisms differ sufficiently to be considered separate species (Jain et al. 2018).

\section{Genomic features related to SQ metabolism}

Genome analysis of Arthrobacter spp. AK01 and AK04 revealed a cluster of genes that were assigned as encoding SQ degradation through the sulfo-EMP pathway (Fig. 3a, b). The prototypical sulfo-EMP pathway was identified in E. coli and involves a 10-gene cluster (yihOPQRSTUV, csqR) (Denger et al. 2014). In E. coli these genes encode a transcription factor (CsqR) (Shimada et al. 2019), putative transmembrane proteins for the import of SQ and export of the end-product of the pathway, DHPS (YihO, YihP). The enzymatic steps involve a sulfoquinovosidase (YihQ) for cleavage of SQ glycosides (Speciale et al. 2016), sulfoquinovose mutarotase (YihR) for interconversion of SQ anomers (Abayakoon et al. 2018b), SQ-sulfofructose (SF) isomerase (YihS) that interconverts SQ, SF and sulforhamnose (Sharma et al. 2021), an ATP-dependent sulfofructose kinase (YihV) that converts SF to SF-1-phosphate (SFP) (Sharma et al. 2021), SFP aldolase (YihT) which converts SFP to SLA and dihydroxyacetone phosphate (Sharma et al. 2021), and an NADH-dependent SLA reductase (YihU) to convert SLA to DHPS (Sharma et al. 2020), which is excreted into the growth media (Fig. 3d).

Both Arthrobacter sp. contained genes encoding SQ-SF isomerase (YihS), SF kinase (YihV), and SFP aldolase (YihT). Consistent with the excretion of SL into the growth media, both strains lacked an SLA reductase homologue, but instead contained an SLA dehydrogenase homologue, annotated as GabD. GabD homologues within sulfoglycolytic gene clusters have been identified for bacteria that utilize the sulfo-ED (Felux et al. 2015; Li et al. 2020) and sulfoSFT (Frommeyer et al. 2020; Liu et al. 2020) pathways, and in Pseudomonas putida SQ1 there is an $\mathrm{NAD}^{+} / \mathrm{NADP}^{+}$dependent SLA dehydrogenase (Felux et al. 2015). Recently, a sulfo-EMP pathway in Bacillus urumqiensis was identified that contained a GabD homologue, SlaB, however, it was unable to be recombinantly expressed and its activity is therefore unknown (Liu et al. 2021); we note that SlaB from Desulfovibrio sp strain DF1 could be recombinantly expressed and was confirmed to be an NADH-dependent, SL-producing SLA dehydrogenase (Burrichter et al. 2018). A proposed pathway for SQ metabolism in these Arthrobacter spp. is shown in Fig. 3c. AK01 and AK04 represent the first characterized examples of sulfoglycolytic bacteria that use a sulfo-EMP pathway but with an SLA dehydrogenase instead of SLA reductase, as described for the prototypical sulfo-EMP pathway in E. coli (Denger et al. 2014). E. coli and related Enterobacteriacae that contain SLA reductases are facultative anaerobes, and the presence of a reducing pathway for excretion of DHPS may support their anaerobic lifestyle. On the other hand, Arthrobacter are normally considered aerobes (Jones and Keddie 2006) (although anaerobic Arthrobacter have been described (Eschbach et al. 2003)), which is consistent with an oxidative pathway for excretion of SL.

SQ import and DHPS/SL export is poorly characterized, but several different strategies have been identified across diverse sulfoglycolytic bacteria. Both AK01 and AK04 contain genes annotated as $\mathrm{ABC}$ transporter cassettes and solute binding proteins. $\mathrm{ABC}$ transporter systems have been identified in Agrobacterium tumefaciens C58 (which uses the sulfo-SMO pathway) (Sharma et al. 2022) and R. leguminosarum SRDI858 (which utilizes a sulfo-ED pathway) (Li et al. 2020). The A. tumefaciens solute binding protein binds SQGro with high affinity (Sharma et al. 2022). We, therefore, propose that these Arthrobacter isolates utilize the solute binding protein and an $\mathrm{ABC}$ transporter system to import SQ or its glycosides. Strain AK04 also contains a TauE permease (a member of the 4-toluene sulfonate uptake permease (TSUP) system) (Shlykov et al. 2012). TSUP proteins are poorly characterized permeases that are suggested to be involved with the transport of sulfur-containing organic compounds. TauE of Cupriavidus necator $\mathrm{H} 16$ is proposed to be involved in the export of sulfolactate (Weinitschke et al. 2007), a function that is consistent with the excretion of SL by AK04. The absence of an obvious permease candidate in the AK01 gene cluster suggests that another protein may adopt this function in this strain.

Other key differences with $E$. coli includes the lack of identifiable SQ mutarotase and SQase encoding genes. Interestingly, the isolated strains could grow on the simple SQ glycoside, methyl $\alpha$-sulfoquinovoside. This suggests that they may harbor an unidentified SQase without homology to known SQases. Finally, both organisms contained a gene encoding an IolG homologue, of unknown function. IolG proteins are 
a)

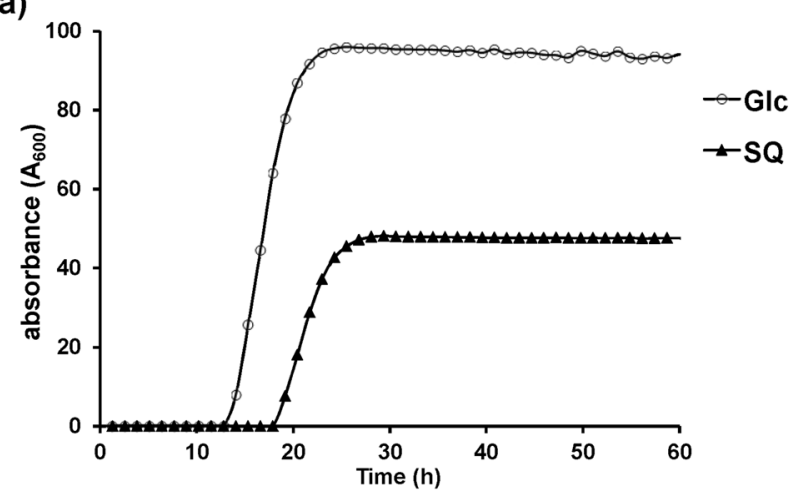

c)

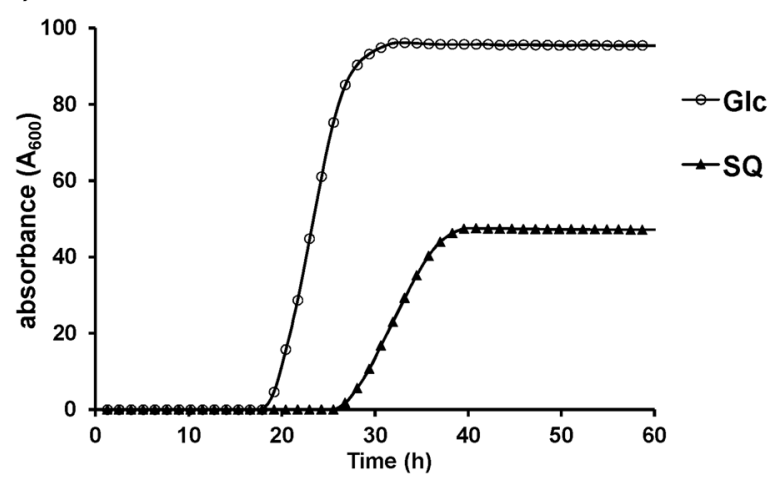

b)

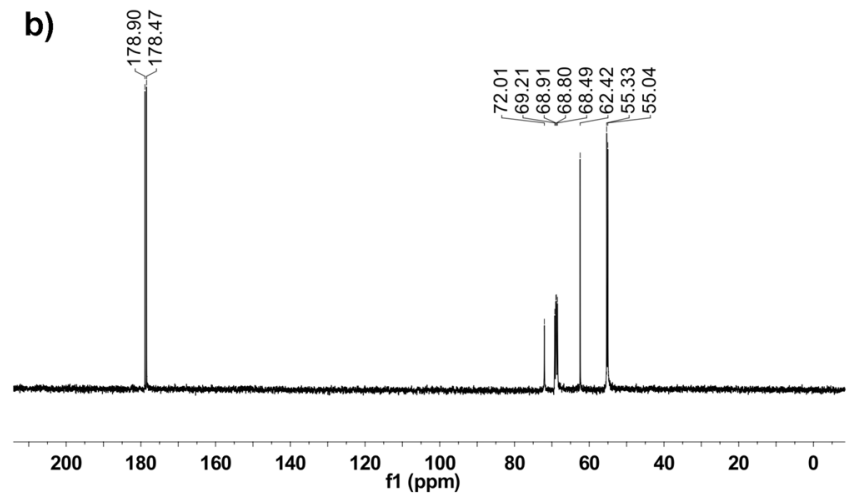

d)

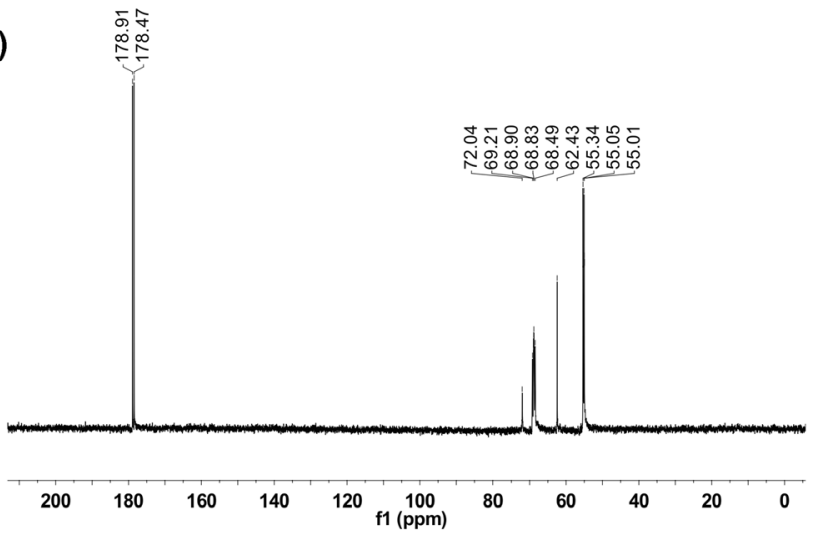

Fig. 3 Growth curves of Arthrobacter strains a AK01 and c AK04 grown on minimal salts media containing 5 mM glucose or SQ. ${ }^{13} \mathrm{C}-\mathrm{NMR}$ $(500 \mathrm{MHz})$ spectra of spent culture media of Arthrobacter strains b AK01 and d AK04 grown on ${ }^{13} \mathrm{C}_{6}-\mathrm{SQ}(7.7 \mathrm{mM})$

NAD(P)-dependent oxidoreductases of the Gfo/Idh/MocA family, and catalyze oxidation of the hydroxyl groups of pyranose and inositol rings (Taberman et al. 2016). Members of this family include inositol dehydrogenase (Idh), which forms 2-keto-myo-inositol (2-inosose) from myo-inositol (Ramaley et al. 1979; Yoshida et al. 2006), glucose-6-phosphate dehydrogenase (G6PD), which forms 6-phosphogluconolactone from glucose-6-phosphate (Rowland et al. 1994), and levoglucosan dehydrogenase, which forms 3-keto-levoglucosan from levoglucosan (1,6-anhydro- $\beta$-D-glucose) (Sugiura et al. 2018; Kuritani et al. 2020). Inositol dehydrogenase IolG from Bacillus subtilis has maximal activity on myo-inositol and possesses activity on D-glucose and D-xylose, and produces D-gluconolactone from the former (Ramaley et al. 1979). Possibly, the Arthobacter spp. IolG homologues may convert SQ to SGL, which coincidentally is an intermediate in the sulfoED pathway (Felux et al. 2015), although the downstream sulfo-ED genes are missing in these organisms. An alternative possibility is suggested by the $\mathrm{Gfo} / \mathrm{Idh} / \mathrm{MocA}$ family member DgpA from the intestinal bacterium PUE. DgpA catalyzes the oxidation of the 3-hydroxyl group of the $\mathrm{C}$-glycoside puerarin, facilitating the elimination of the aglycon and formation of 3-keto-2-hydroxyglucal (1,5-anhydro-D-erythro-hex-1-en-3ulose) (Nakamura et al. 2020). A similar process applied to an SQ glycoside could facilitate the cleavage of the glycoside in the absence of an SQase and would give rise to the corresponding 6-sulfo-3-keto-2-hydroxyglucal, the fate of which is uncertain. However, we were unable to identify possible companion genes encoding proteins that would be required to enable the elimination/isomerization/reduction to SQ.

A search for organisms with gene clusters related to strains AK01 and AK04 led to identification of other Arthrobacter strains with syntenic or closely related sulfo-EMP gene clusters (Fig. 4). Other Arthrobacter spp. were identified that contained gene clusters with architectures identical to AK01; and both Arthrobacter spp. and Pseudarthrobacter spp. were identified with gene clusters identical to AK04. Non-identical but closely syntenic sulfo-EMP gene clusters were observed in select Actinobacteria including other Arthrobacter spp. and Microbacterium sp. No. 7 (both order Micrococcales), and a more distantly related cluster in the actinobacteria Acrocarpospora corrugata (order Streptosporangiales) and Phytohabitans houttuyneae (order Micromonosporales). 


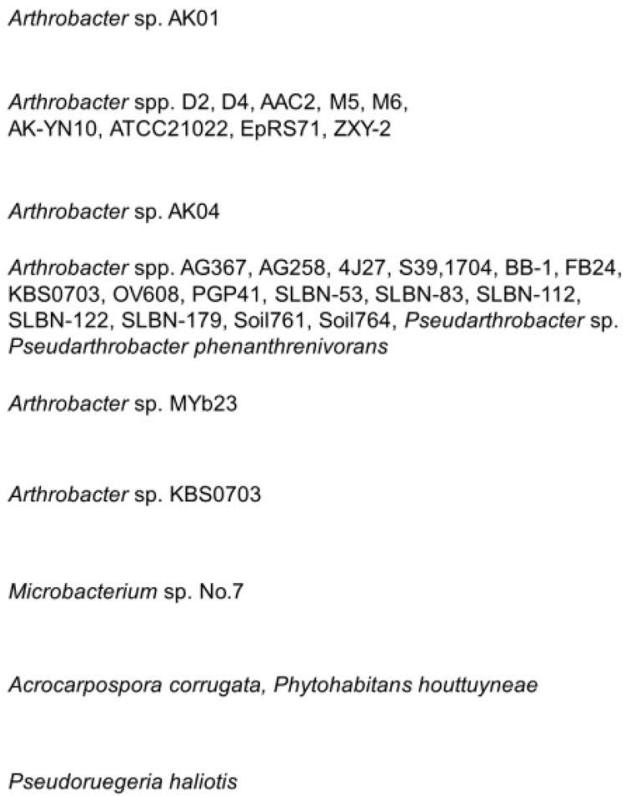

- GabD (SLA dehydrogenase)

SFP aldolase

SF Kinase

- ABC transporter permease

transcriptional regulator

$A B C$ transporter substrate binding protein

SQ isomerase

- oxidoreductase

- TauE permease
Fig. 4 Distribution and architecture of sulfo-EMP gene clusters in Arthrobacter and related organisms. Syntenic relationship of sulfo-EMP gene clusters in Arthrobacter sp. AK01 and AK04 with homologous gene clusters. Colored links indicate $\geq 30 \%$ protein sequence similarity. Genome accession codes: Arthrobacter sp. D2 (LUKB01000109.1), Arthrobacter sp. D4 (LUKC01000078.1), Arthrobacter sp. AAC2 (JAAGBD010000014.1), Arthrobacter sp. M5 (LVCB01000107.1), Arthrobacter sp. M6 (LVCC01000103.1), Arthrobacter sp. AK-YN10 (AVPD02000157.1), Arthrobacter sp. ATCC 21,022 (CP014196.1) Arthrobacter sp. EpRS71 (LNUV01000003.1), Arthrobacter sp. ZXY-2 (CP017421.1), Arthrobacter sp. AG367 (VIVE01000010.1), Arthrobacter sp. AG258 (SOBI01000009.1), Arthrobacter sp. 4J27 (CAQI01000048.1), Arthrobacter sp. S39 (SIHX01000007.1), Arthrobacter sp.1704 (SOBD01000016.1), Arthrobacter sp. BB-1 (VDEV01000010.1), Arthrobacter sp. FB24 (CP000454.1),

\section{Conclusions}

Two sulfoglycolytic soil bacteria belonging to the Arthrobacter genus (strains AK01 and AK04) were isolated from soil by enrichment culture involving growth on SQ. The stationary phase optical density of these bacteria when grown on SQ was approximately half that of growth on glucose, consistent with utilization of only three of the six carbons of SQ. Both possessed a variant of the sulfo-EMP pathway that uses an SLA dehydrogenase to produce SL that is secreted into the growth media, and which is proposed to arise from an SLA dehydrogenase (GabD). SL in turn becomes available for other members of the microbial community that specialize in its metabolism (Rein et al. 2005; Cook et al. 2006; Denger et al. 2009, 2012; Denger and Cook 2010). Prior to this work, the only sulfo-EMP pathway bacteria that have been characterized were a range of
Arthrobacter sp. KBS0703 (MVDG02000001.1), Arthrobacter sp. OV608 (FOEZ01000003.1), Arthrobacter sp. PGP41 (CP026514.1), Arthrobacter sp. SLBN-53 (VFMZ01000001.1), Arthrobacter sp. SLBN-83 (VFMX01000001.1), Arthrobacter sp. SLBN-112 (VFMU01000001.1), Arthrobacter sp. SLBN-122 (VFMS01000001.1), Arthrobacter sp. SLBN-179 (VFNR01000001.1), Arthrobacter sp. Soil761 (LMSF01000007.1), Arthrobacter sp. Soil764 (LMSI01000008.1), Pseudarthrobacter phenanthrenivorans (CP002379.1), Pseudarthrobacter phenanthrenivorans (RBNH01000003.1), Pseudarthrobacter phenanthrenivorans (VHJD01000009.1), Pseudarthrobacter sp. AG30 (QEHL01000024.1), Arthrobacter sp. MYb23 (PCPR01000010.1), Arthrobacter sp. KBS0703 (MVDG02000001.1), Microbacterium sp. No. 7 (CP012697.1), Acrocarpospora corrugata (BLAD01000050.1), Phytohabitans houttuyneae (BLPF01000004.1), Pseudoruegeria haliotis (PVTD01000003.1)

E. coli strains, which produce DHPS through the action of SLA reductase (Denger et al. 2014). Notably, strains AK01 and AK04 lack genes assigned as encoding an SQase, but nonetheless could grow on MeSQ, indicating the presence of a non-specific SQase, a novel SQase that is not readily identified by sequence homology, or a novel lyase pathway. This study highlights the core genes required for sulfoglycolysis (YihS, YihT, YihU, YihV) and constitutes the first examples of sulfo-EMP bacteria isolated from soil.

Supplementary Information The online version contains supplementary material available at https://doi.org/10.1007/s00203-022-02803-2.

Author contributions SJW conceived the study with input from SP. AK and $\mathrm{PvdP}$ performed enrichment culture and microbial characterization. $\mathrm{JM}$ synthesized ${ }^{13} \mathrm{C}_{6}$-SQ. AK and JM performed bioinformatic analysis. $\mathrm{MH}$ and SP performed genome sequencing, assembly and analysis. AK and SJW wrote the paper with input and approval of the final draft. 
Funding Open Access funding enabled and organized by CAUL and its Member Institutions. This work was supported by the Australian Research Council (DP210100233, DP210100235).

Availability of data and materials The datasets generated and/ or analyzed during the current study are available in the GenBank database with accession codes SAMN23041292 (strain AK01) and SAMN23041293 (strain AK04)

Code availability Scripts used for bioinformatic search pipeline are available on the GitHub repository, https://github.com/jmui-unimelb/ Gene-Cluster-Search-Pipeline.

\section{Declarations}

Conflict of interest The authors declare that they have no conflict of interest.

Ethics approval Not applicable.

Consent to participate Not applicable.

Consent for publication Not applicable.

Open Access This article is licensed under a Creative Commons Attribution 4.0 International License, which permits use, sharing, adaptation, distribution and reproduction in any medium or format, as long as you give appropriate credit to the original author(s) and the source, provide a link to the Creative Commons licence, and indicate if changes were made. The images or other third party material in this article are included in the article's Creative Commons licence, unless indicated otherwise in a credit line to the material. If material is not included in the article's Creative Commons licence and your intended use is not permitted by statutory regulation or exceeds the permitted use, you will need to obtain permission directly from the copyright holder. To view a copy of this licence, visit http://creativecommons.org/licenses/by/4.0/.

\section{References}

Abayakoon P et al (2018a) Structural and biochemical insights into the function and evolution of sulfoquinovosidases. ACS Cent Sci 4:1266-1273. https://doi.org/10.1021/acscentsci.8b00453

Abayakoon P et al (2018b) Discovery and characterization of a sulfoquinovose mutarotase using kinetic analysis at equilibrium by exchange spectroscopy. Biochem J 475:1371-1383. https://doi. org/10.1042/bcj20170947

Benson AA, Shibuya I (1961) Sulfocarbohydrate metabolism. Fed Proc 20:79

Benson AA, Daniel H, Wiser R (1959) A sulfolipid in plants. Proc Natl Acad Sci USA 45:1582-1587. https://doi.org/10.1073/pnas. 45.11.1582

Burrichter A et al (2018) Anaerobic degradation of the plant sugar sulfoquinovose concomitant with $\mathrm{H} 2 \mathrm{~S}$ production: Escherichia coli K-12 and Desulfovibrio sp. strain DF1 as co-culture model. Front Microbiol. 9. https://doi.org/10.3389/fmicb.2018.02792

Cook AM, Denger K, Smits TH (2006) Dissimilation of C3-sulfonates. Arch Microbiol 185:83-90. https://doi.org/10.1007/ s00203-005-0069-1

Denger K, Cook AM (2010) Racemase activity effected by two dehydrogenases in sulfolactate degradation by Chromohalobacter salexigens: purification of (S)-sulfolactate dehydrogenase.
Microbiology 156:967-974. https://doi.org/10.1099/mic.0. 034736-0

Denger K, Mayer J, Buhmann M, Weinitschke S, Smits TH, Cook AM (2009) Bifurcated degradative pathway of 3-sulfolactate in Roseovarius nubinhibens ISM via sulfoacetaldehyde acetyltransferase and $(S)$-cysteate sulfolyase. J Bacteriol 191:5648-5656. https:// doi.org/10.1128/jb.00569-09

Denger K, Huhn T, Hollemeyer K, Schleheck D, Cook AM (2012) Sulfoquinovose degraded by pure cultures of bacteria with release of $\mathrm{C}_{3}$-organosulfonates: complete degradation in two-member communities. FEMS Microbiol Lett 328:39-45. https://doi.org/ 10.1111/j.1574-6968.2011.02477.x

Denger K et al (2014) Sulphoglycolysis in Escherichia coli K-12 closes a gap in the biogeochemical sulphur cycle. Nature 507:114-117. https://doi.org/10.1038/nature12947

Eschbach M, Möbitz H, Rompf A, Jahn D (2003) Members of the genus Arthrobacter grow anaerobically using nitrate ammonification and fermentative processes: anaerobic adaptation of aerobic bacteria abundant in soil. FEMS Microbiol Lett 223:227-230. https://doi.org/10.1016/S0378-1097(03)00383-5\%JFEMSMicro biologyLetters

Felux AK, Spiteller D, Klebensberger J, Schleheck D (2015) EntnerDoudoroff pathway for sulfoquinovose degradation in $\mathrm{Pseu}$ domonas putida SQ1. Proc Natl Acad Sci USA 112:E4298-4305. https://doi.org/10.1073/pnas.1507049112

Frommeyer B et al (2020) Environmental and intestinal phylum firmicutes bacteria metabolize the plant sugar sulfoquinovose via a 6-deoxy-6-sulfofructose transaldolase pathway. Science 23:101510. https://doi.org/10.1016/j.isci.2020.101510

Goddard-Borger ED, Williams SJ (2017) Sulfoquinovose in the biosphere: occurrence, metabolism and functions. Biochem J 474:827-849. https://doi.org/10.1042/BCJ20160508

Harwood JL, Nicholls RG (1979) The plant sulpholipid—a major component of the sulphur cycle. Biochem Soc Trans 7:440-447

Jain C, Rodriguez-R LM, Phillippy AM, Konstantinidis KT, Aluru S (2018) High throughput ANI analysis of $90 \mathrm{~K}$ prokaryotic genomes reveals clear species boundaries. Nat Commun 9:5114. https://doi.org/10.1038/s41467-018-07641-9

Jones D, Keddie RM (2006) The Genus Arthrobacter. In: Dworkin M, Falkow S, Rosenberg E, Schleifer K-H, Stackebrandt E (eds) The Prokaryotes: volume 3: archaea. Bacteria: firmicutes, actinomycete. Springer, New York, NY, pp 945-960

Kim M, Oh HS, Park SC, Chun J (2014) Towards a taxonomic coherence between average nucleotide identity and 16S rRNA gene sequence similarity for species demarcation of prokaryotes. Int J Syst Evol Microbiol 64:346-351. https://doi.org/10.1099/ijs.0. 059774-0

Kuritani Y et al (2020) Conversion of levoglucosan into glucose by the coordination of four enzymes through oxidation, elimination, hydration, and reduction. Sci Rep 10:20066. https://doi.org/10. 1038/s41598-020-77133-8

Li J et al (2020) A sulfoglycolytic Entner-Doudoroff pathway in Rhizobium leguminosarum bv. trifolii SRDI565. Appl Environ Microbiol 86:e00750-e00720. https://doi.org/10.1128/aem.00750-20

Liu Y, Wei Y, Zhou Y, Ang EL, Zhao H, Zhang Y (2020) A transaldolase-dependent sulfoglycolysis pathway in Bacillus megaterium DSM 1804. Biochem Biophys Res Commun 533:1109-1114. https://doi.org/10.1016/j.bbrc.2020.09.124

Liu J et al (2021) Mechanistically diverse pathways for sulfoquinovose degradation in bacteria. ACS Catal 11:14740-14750. https://doi. org/10.1021/acscatal.1c04321

Nakamura K, Zhu S, Komatsu K, Hattori M, Iwashima M (2020) Deglycosylation of the isoflavone $\mathrm{C}$-glucoside puerarin by a combination of two recombinant bacterial enzymes and 3-oxo-glucose. Appl Environ Microbiol. https://doi.org/10.1128/aem.00607-20 
Ramaley R, Fujita Y, Freese E (1979) Purification and properties of Bacillus subtilis inositol dehydrogenase. J Biol Chem 254:7684-7690

Rein U, Gueta R, Denger K, Ruff J, Hollemeyer K, Cook AM (2005) Dissimilation of cysteate via 3-sulfolactate sulfo-lyase and a sulfate exporter in Paracoccus pantotrophus NKNCYSA. Microbiology 151:737-747. https://doi.org/10.1099/mic.0.27548-0

Rowland P, Basak AK, Gover S, Levy HR, Adams MJ (1994) The three-dimensional structure of glucose 6-phosphate dehydrogenase from Leuconostoc mesenteroides refined at 2.0 A resolution. Structure 2:1073-1087. https://doi.org/10.1016/S0969-2126(94) 00110-3

Seemann T (2014) Prokka: rapid prokaryotic genome annotation. Bioinformatics 30:2068-2069. https://doi.org/10.1093/bioinforma tics/btu 153

Sharma M et al (2020) Dynamic structural changes accompany the production of dihydroxypropanesulfonate by sulfolactaldehyde reductase. ACS Catal 10:2826-2836. https://doi.org/10.1021/ acscatal.9b04427

Sharma M et al (2021) Molecular basis of sulfosugar selectivity in sulfoglycolysis. ACS Cent Sci 7:476-487. https://doi.org/10.1021/ acscentsci.0c01285

Sharma M, Lingford JP, Petricevic M, Snow AJP, Zhang Y, Jarva M, Mui JW-Y, Scott NE, Saunders EC, Mao R, Epa R, da Silva BM, Pires DEV, Ascher DB, McConville MJ, Davies GJ, Williams SJ, Goddard-Borger ED (2022) Oxidative desulfurization pathway for complete catabolism of sulfoquinovose by bacteria. Proc Natl Acad Sci USA 119:e2116022119. https://doi.org/10.1073/pnas. 2116022119

Shimada T, Yamamoto K, Nakano M, Watanabe H, Schleheck D, Ishihama A (2019) Regulatory role of CsqR (YihW) in transcription of the genes for catabolism of the anionic sugar sulfoquinovose (SQ) in Escherichia coli K-12. Microbiology 165:78-89. https:// doi.org/10.1099/mic.0.000740

Shlykov MA, Zheng WH, Chen JS, Saier MH Jr (2012) Bioinformatic characterization of the 4-toluene sulfonate uptake permease (TSUP) family of transmembrane proteins. Biochim Biophys Acta 1818:703-717. https://doi.org/10.1016/j.bbamem.2011.12.005
Snow AJD, Burchill L, Sharma M, Davies GJ, Williams SJ (2021) Sulfoglycolysis: catabolic pathways for metabolism of sulfoquinovose. Chem Soc Rev 50:13628-13645. https://doi.org/10.1039/ D1CS00846C

Speciale G, Jin Y, Davies GJ, Williams SJ, Goddard-Borger ED (2016) YihQ is a sulfoquinovosidase that cleaves sulfoquinovosyl diacylglyceride sulfolipids. Nat Chem Biol 12:215-217. https://doi.org/ 10.1038/nchembio.2023

Sugiura M, Nakahara M, Yamada C, Arakawa T, Kitaoka M, Fushinobu S (2018) Identification, functional characterization, and crystal structure determination of bacterial levoglucosan dehydrogenase. J Biol Chem 293:17375-17386. https://doi.org/10.1074/ jbc.RA118.004963

Taberman H, Parkkinen T, Rouvinen J (2016) Structural and functional features of the NAD(P) dependent Gfo/Idh/MocA protein family oxidoreductases. Protein Sci 25:778-786. https://doi.org/10. 1002/pro.2877

Weinitschke S, Denger K, Cook AM, Smits THM (2007) The DUF81 protein TauE in Cupriavidus necator $\mathrm{H} 16$, a sulfite exporter in the metabolism of C2 sulfonates. Microbiology 153:3055-3060. https://doi.org/10.1099/mic.0.2007/009845-0

Yoon SH, Ha SM, Lim J, Kwon S, Chun J (2017) A large-scale evaluation of algorithms to calculate average nucleotide identity. Antonie Van Leeuwenhoek 110:1281-1286. https://doi.org/10. 1007/s10482-017-0844-4

Yoshida K, Yamaguchi M, Morinaga T, Ikeuchi M, Kinehara M, Ashida H (2006) Genetic modification of Bacillus subtilis for production of D-chiro-inositol, an investigational drug candidate for treatment of type 2 diabetes and polycystic ovary syndrome. Appl Environ Microbiol 72:1310-1315. https://doi.org/10.1128/ aem.72.2.1310-1315.2006

Publisher's Note Springer Nature remains neutral with regard to jurisdictional claims in published maps and institutional affiliations. 